\title{
On the Retrieval of the Directional Scattering Matrix from Directional Noise*
}

\author{
Kees Wapenaar ${ }^{\dagger}$ and Jan Thorbecke ${ }^{\dagger}$
}

\begin{abstract}
The crosscorrelation of ambient acoustic noise observed at two receivers yields the impulse response between these receivers, assuming that the noise field is diffuse. In practical situations the noise field exhibits directionality, which imprints the angle-dependent correlation function. For the situation of a directional scatterer in a directional noise field, the correlation function contains the product of the directional scattering matrix and the directional noise. This seemingly underdetermined problem can be resolved by exploiting a relation between the causal and acausal parts of the correlation function. For a given pair of receivers, the causal and acausal parts of the correlation function contain the same element of the scattering matrix (by reciprocity) but different elements of the directional noise field. This property can be used to estimate the directionality of the noise (apart from an undetermined scaling factor) and, subsequently, of the scattering matrix.
\end{abstract}

Key words. scattering matrix, optical theorem, ambient noise

AMS subject classifications. 70Sxx, 74J20, 81Uxx, 86A15

DOI. $10.1137 / 12086131 X$

1. Introduction. Recently, methodologies have been developed to retrieve a medium's acoustic impulse response from ambient noise $[1,2,3,4,5,6]$. Assuming the noise field is diffuse, the crosscorrelation of the responses at two receivers converges to the Green's function between these receivers. This principle has been successfully applied in the fields of ultrasonics [7], regional seismology [8, 9, 10, 11], exploration seismic [12], underwater acoustics [13, 14], and medical imaging [15]. For a review of the theory and the many applications see [16], [17], [18], and [19].

In many practical situations, the noise field is not purely diffuse but exhibits directionality $[20,21,22,23,24,25]$. This causes artificial directionality of the retrieved Green's function. In addition, it may lead to spurious events because of incomplete cancellation of specific stationary-phase contributions. Several methods have been proposed to compensate for the directionality of the noise field, ranging from correlating the coda of the correlation $[26,27,28]$ and directional balancing [29] to multidimensional deconvolution [30]. These methods apply to different configurations and each of them yield more accurate Green's functions than the crosscorrelation method in those configurations.

Here we present a method to retrieve the directional scattering matrix of a scattering object from directional noise. The configuration consists of a scatterer with compact support in a homogeneous embedding medium. The proposed method is based on the following principle;

\footnotetext{
${ }^{*}$ Received by the editors January 4, 2012; accepted for publication (in revised form) November 15, 2012; published electronically February 12, 2013. This work was supported by the Netherlands Research Centre for Integrated Solid Earth Science (ISES).

http://www.siam.org/journals/siims/6-1/86131.html

${ }^{\dagger}$ Department of Geoscience and Engineering, Delft University of Technology, Delft 2600 GA, Netherlands (c.p.a.wapenaar@tudelft.nl, j.w.thorbecke@tudelft.nl).
} 


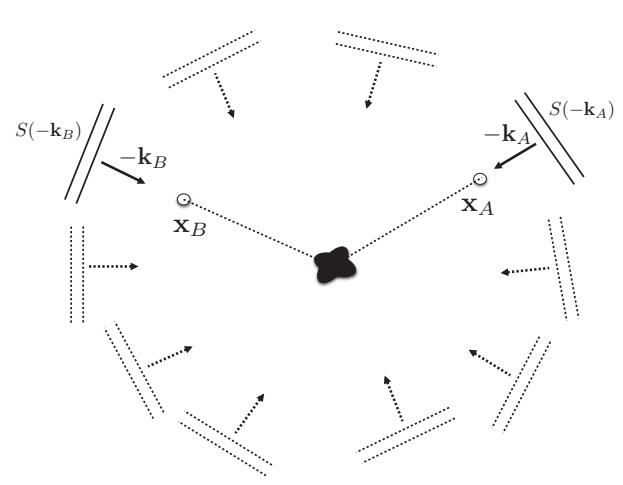

Figure 1. A scatterer with compact support in a homogeneous embedding medium, illuminated by nondiffuse noise. For the scattered field, the causal and acausal parts of the crosscorrelation of the noise at two receivers $\mathbf{x}_{A}$ and $\mathbf{x}_{B}$ are dominated by waves propagating in opposite directions through the receivers and the scatterer. The spectral ratio of the time-reversed acausal part and the causal part gives the ratio of the noise spectra corresponding to these directions (because the scattering function is the same in both directions).

see also Figure 1. For the scattered field, the causal part of the crosscorrelation of the noise at two receivers is dominated by the wave propagating through one of the receivers via the scatterer to the other receiver, whereas the acausal part is dominated by the wave propagating in the opposite direction. Because of reciprocity, both parts of the correlation function are related to the same element of the scattering matrix, but they are weighted by different noise spectra. The spectral division of the time-reversed acausal part and the causal part of the correlation function gives the ratio of these noise spectra. This ratio can be used to compensate for the imprint of the directional noise on the correlation function. Apart from an unknown constant scaling factor, this yields the correct directional properties of the scattering matrix. This method distinguishes itself from other methods that compensate for noise directionality by explicitly exploiting the relation between the causal and acausal parts of the correlation function. Moreover, in principle the method can be applied on a receiver-by-receiver basis, but applying it to an array of receivers stabilizes the result.

The outline of this paper is as follows. In section 2 we discuss the model for the response of a directional scatterer to directional noise. In section 3 we carefully evaluate the correlation of the responses at two receiver positions. In section 4 we discuss some properties of this correlation function. Based on this, in section 5 we show how the noise directivity and, subsequently, the directional scattering matrix can be retrieved from the correlation function. We end with concluding remarks in section 6 .

\section{The data model.}

2.1. Three-dimensional situation. We consider a scatterer with compact support around the origin, embedded in an otherwise homogeneous medium with propagation velocity $c$. For this configuration, the far-field response of a monochromatic plane wave with unit amplitude is given by [31]

$$
\psi(\mathbf{x}, \mathbf{k})=\psi^{i}(\mathbf{x}, \mathbf{k})+\psi^{s}(\mathbf{x}, \mathbf{k})
$$

Copyright (c) by SIAM. Unauthorized reproduction of this article is prohibited. 
where $\psi^{i}(\mathbf{x}, \mathbf{k})$ and $\psi^{s}(\mathbf{x}, \mathbf{k})$ are the incident and scattered fields, respectively, according to

$$
\psi^{i}(\mathbf{x}, \mathbf{k})=\exp (i \mathbf{k} \cdot \mathbf{x})
$$

( $i$ is the imaginary unit) and

$$
\psi^{s}(\mathbf{x}, \mathbf{k})=f\left(\mathbf{k}_{x}, \mathbf{k}\right) \exp (i k|\mathbf{x}|) /|\mathbf{x}| .
$$

Here $f\left(\mathbf{k}_{x}, \mathbf{k}\right)$ is the scattering matrix of the scatterer, where $\mathbf{k}$ and $\mathbf{k}_{x}$ are the wave vectors of the incident and scattered fields, respectively. Note that $|\mathbf{k}|=|k|$ with wavenumber $k=\omega / c$, where $\omega$ is the angular frequency. (k can have any direction.) Moreover, $\mathbf{k}_{x}=k \mathbf{x} /|\mathbf{x}|$, where $\mathbf{x}$ is the Cartesian coordinate vector of the observation point. The scattering matrix accounts for nonlinear (primary and multiple) scattering within the scatterer. The far-field approximation (3) holds when

$$
|\mathbf{x}| \gg \frac{a^{2}}{\lambda}=\frac{a^{2}|\mathbf{k}|}{2 \pi}
$$

where $\lambda$ is the wavelength in the homogeneous embedding medium and $a$ the effective size of the scatterer (i.e., the support of the inverse spatial Fourier transform of the scattering matrix $f[32])$. The scattering matrix obeys the reciprocity relation [31]

$$
f\left(\mathbf{k}_{x}, \mathbf{k}\right)=f\left(-\mathbf{k},-\mathbf{k}_{x}\right)
$$

and the generalized optical theorem [31, 33, 34, 35]

$$
\frac{1}{2 i}\left\{f\left(\mathbf{k}^{\prime}, \mathbf{k}\right)-f^{*}\left(\mathbf{k}, \mathbf{k}^{\prime}\right)\right\}=\frac{k}{4 \pi} \oint f\left(\mathbf{k}^{\prime \prime}, \mathbf{k}\right) f^{*}\left(\mathbf{k}^{\prime \prime}, \mathbf{k}^{\prime}\right) \mathrm{d} \Omega_{k^{\prime \prime}},
$$

where the superscript asterisk denotes complex conjugation and $\mathrm{d} \Omega_{k^{\prime \prime}}=\mathrm{d}^{2} \mathbf{k}^{\prime \prime} /\left|\mathbf{k}^{\prime \prime}\right|^{2}$.

In the following we consider a directional noise field, consisting of a distribution of plane waves with time-dependent noise functions $N(\mathbf{n}, t)$, where $t$ denotes time and $\mathbf{n}$ is a normal vector, indicating the propagation direction of the plane wave. The noise functions for different propagation directions are mutually uncorrelated, according to

$$
\left\langle N\left(\mathbf{n}^{\prime}, t\right) * N(\mathbf{n},-t)\right\rangle=S(\mathbf{n}, t) \delta\left(\mathbf{n}-\mathbf{n}^{\prime}\right) .
$$

The inline asterisk denotes temporal convolution, but the time-reversal of the second noise function turns the convolution into a correlation. The notation $\langle\cdot\rangle$ denotes ensemble averaging, which is in practice replaced by averaging over sufficiently long time. $S(\mathbf{n}, t)$ is the autocorrelation of the noise function of the plane wave with direction $\mathbf{n}$, and $\delta\left(\mathbf{n}-\mathbf{n}^{\prime}\right)$ is the Dirac delta function, defined on a sphere with unit radius (because $|\mathbf{n}|=\left|\mathbf{n}^{\prime}\right|=1$ ). Note that $S(\mathbf{n}, t)$ quantifies the directionality of the noise field. We define the temporal Fourier transform $H(\omega)$ of a time-dependent function $H(t)$ as

$$
H(\omega)=\int_{-\infty}^{\infty} H(t) \exp (i \omega t) \mathrm{d} t
$$

Copyright (C) by SIAM. Unauthorized reproduction of this article is prohibited. 
In this paper we will not use different symbols for time- and frequency-domain functions. (There can be no confusion because we always write the arguments between brackets.) With this definition, the Fourier transform of (7) becomes

$$
\left\langle N\left(\mathbf{n}^{\prime}, \omega\right) N^{*}(\mathbf{n}, \omega)\right\rangle=S(\mathbf{n}, \omega) \delta\left(\mathbf{n}-\mathbf{n}^{\prime}\right),
$$

which we write more compactly as

$$
\left\langle N\left(\mathbf{k}^{\prime}\right) N^{*}(\mathbf{k})\right\rangle=S(\mathbf{k})|\mathbf{k}|^{2} \delta\left(\mathbf{k}-\mathbf{k}^{\prime}\right)
$$

with $\mathbf{k}=\mathbf{n} k$ and $\mathbf{k}^{\prime}=\mathbf{n}^{\prime} k$. Here $\delta\left(\mathbf{k}-\mathbf{k}^{\prime}\right)$ is defined on a sphere with radius $|\mathbf{k}|=\left|\mathbf{k}^{\prime}\right|$. Note that $S(\mathbf{k})$ is the power spectrum of the noise for a plane wave with wave vector $\mathbf{k}$.

Using the superposition principle, the response to the distribution of plane waves is in the space-frequency domain written as

$$
\Psi(\mathbf{x}, \omega)=\oint \psi(\mathbf{x}, \mathbf{k}) N(\mathbf{k}) \mathrm{d} \Omega_{k}
$$

with $\psi(\mathbf{x}, \mathbf{k})$ defined in (1)-(3).

2.2. Two-dimensional situation. For the two-dimensional (2D) situation, most of the equations in section 2.1 hold but with $2 \mathrm{D}$ coordinate and wave vectors. However, because Green's functions are different in two dimensions, the scattered field in (3) needs to be replaced by

$$
\psi^{s}(\mathbf{x}, \mathbf{k})=f\left(\mathbf{k}_{x}, \mathbf{k}\right) \frac{\exp (i k|\mathbf{x}|+i \mu \pi / 4)}{\sqrt{|k||\mathbf{x}|}}, \quad \mu=\operatorname{sign}(k) .
$$

Moreover, the generalized optical theorem in (6) needs to be replaced by

$$
\frac{1}{2 i}\left\{f\left(\mathbf{k}^{\prime}, \mathbf{k}\right)-f^{*}\left(\mathbf{k}, \mathbf{k}^{\prime}\right)\right\}=\frac{\mu}{\sqrt{8 \pi}} \oint f\left(\mathbf{k}^{\prime \prime}, \mathbf{k}\right) f^{*}\left(\mathbf{k}^{\prime \prime}, \mathbf{k}^{\prime}\right) \mathrm{d} \Omega_{k^{\prime \prime}}
$$

with $\mathrm{d} \Omega_{k^{\prime \prime}}=\mathrm{d} \mathbf{k}^{\prime \prime} /\left|\mathbf{k}^{\prime \prime}\right|$ and (10) needs to be replaced by

$$
\left\langle N\left(\mathbf{k}^{\prime}\right) N^{*}(\mathbf{k})\right\rangle=S(\mathbf{k})|\mathbf{k}| \delta\left(\mathbf{k}-\mathbf{k}^{\prime}\right)
$$

with $\delta\left(\mathbf{k}-\mathbf{k}^{\prime}\right)$ defined on a circle with radius $|\mathbf{k}|=\left|\mathbf{k}^{\prime}\right|$.

\section{The correlation function.}

3.1. 3D situation. We define the crosscorrelation of the responses at two receiver positions $\mathbf{x}_{A}$ and $\mathbf{x}_{B}$ as

$$
C\left(\mathbf{x}_{B}, \mathbf{x}_{A}, t\right)=\left\langle\Psi\left(\mathbf{x}_{B}, t\right) * \Psi\left(\mathbf{x}_{A},-t\right)\right\rangle .
$$

In the frequency domain this becomes

$$
C\left(\mathbf{x}_{B}, \mathbf{x}_{A}, \omega\right)=\left\langle\Psi\left(\mathbf{x}_{B}, \omega\right) \Psi^{*}\left(\mathbf{x}_{A}, \omega\right)\right\rangle .
$$


Upon substitution of (11) we obtain, using (10),

$$
\begin{aligned}
C\left(\mathbf{x}_{B}, \mathbf{x}_{A}, \omega\right) & =\left\langle\oint \oint \psi\left(\mathbf{x}_{B}, \mathbf{k}^{\prime}\right) N\left(\mathbf{k}^{\prime}\right) \psi^{*}\left(\mathbf{x}_{A}, \mathbf{k}\right) N^{*}(\mathbf{k}) \mathrm{d} \Omega_{k} \mathrm{~d} \Omega_{k^{\prime}}\right\rangle \\
& =\oint \psi\left(\mathbf{x}_{B}, \mathbf{k}\right) \psi^{*}\left(\mathbf{x}_{A}, \mathbf{k}\right) S(\mathbf{k}) \mathrm{d} \Omega_{k} .
\end{aligned}
$$

Substitution of (1) gives

$$
C\left(\mathbf{x}_{B}, \mathbf{x}_{A}, \omega\right)=\mathcal{L}\left(\psi_{B}^{i}, \psi_{A}^{i}\right)+\mathcal{L}\left(\psi_{B}^{s}, \psi_{A}^{i}\right)+\mathcal{L}\left(\psi_{B}^{i}, \psi_{A}^{s}\right)+\mathcal{L}\left(\psi_{B}^{s}, \psi_{A}^{s}\right),
$$

where the form $\mathcal{L}\left(\psi_{B}, \psi_{A}\right)$ is defined as

$$
\mathcal{L}\left(\psi_{B}, \psi_{A}\right) \doteq \oint \psi\left(\mathbf{x}_{B}, \mathbf{k}\right) \psi^{*}\left(\mathbf{x}_{A}, \mathbf{k}\right) S(\mathbf{k}) \mathrm{d} \Omega_{k}
$$

We evaluate the right-hand side of (18) term by term [36]. For the first term we obtain, using $(2)$

$$
\begin{aligned}
\mathcal{L}\left(\psi_{B}^{i}, \psi_{A}^{i}\right) & =\oint \psi^{i}\left(\mathbf{x}_{B}, \mathbf{k}\right)\left\{\psi^{i}\left(\mathbf{x}_{A}, \mathbf{k}\right)\right\}^{*} S(\mathbf{k}) \mathrm{d} \Omega_{k} \\
& =\oint \exp \left(i \mathbf{k} \cdot\left(\mathbf{x}_{B}-\mathbf{x}_{A}\right)\right) S(\mathbf{k}) \mathrm{d} \Omega_{k}
\end{aligned}
$$

The main contributions come from $\mathbf{k}$-values for which the argument of the exponential is stationary [5]. This occurs when $\mathbf{k}$ is parallel with $\mathbf{x}_{B}-\mathbf{x}_{A}$, i.e., for $\mathbf{k}= \pm \mathbf{k}_{A B}$ with $\mathbf{k}_{A B}=$ $k\left(\mathbf{x}_{B}-\mathbf{x}_{A}\right) /\left|\mathbf{x}_{B}-\mathbf{x}_{A}\right| ;$ see Figure 2(a). Using the method of stationary phase (Appendix A.1), we find for large $|k|$

$$
\mathcal{L}\left(\psi_{B}^{i}, \psi_{A}^{i}\right)=\frac{2 \pi}{i k\left|\mathbf{x}_{B}-\mathbf{x}_{A}\right|}\left[\exp \left(i k\left|\mathbf{x}_{B}-\mathbf{x}_{A}\right|\right) S\left(\mathbf{k}_{A B}\right)-\exp \left(-i k\left|\mathbf{x}_{B}-\mathbf{x}_{A}\right|\right) S\left(-\mathbf{k}_{A B}\right)\right]
$$

or

$$
\mathcal{L}\left(\psi_{B}^{i}, \psi_{A}^{i}\right)=\frac{8 \pi^{2}}{i k}\left[\bar{G}\left(\mathbf{x}_{B}, \mathbf{x}_{A}, \omega\right) S\left(\mathbf{k}_{A B}\right)-\bar{G}^{*}\left(\mathbf{x}_{B}, \mathbf{x}_{A}, \omega\right) S\left(-\mathbf{k}_{A B}\right)\right],
$$

where $\bar{G}\left(\mathbf{x}_{B}, \mathbf{x}_{A}, \omega\right)$ is the Green's function in the homogeneous embedding medium, defined as

$$
\bar{G}\left(\mathbf{x}_{B}, \mathbf{x}_{A}, \omega\right)=\frac{\exp \left(i k\left|\mathbf{x}_{B}-\mathbf{x}_{A}\right|\right)}{4 \pi\left|\mathbf{x}_{B}-\mathbf{x}_{A}\right|} .
$$

This is the Fourier transform of

$$
\bar{G}\left(\mathbf{x}_{B}, \mathbf{x}_{A}, t\right)=\frac{\delta\left(t-t_{\text {dir }}\right)}{4 \pi\left|\mathbf{x}_{B}-\mathbf{x}_{A}\right|}
$$

with $t_{\text {dir }}=\left|\mathbf{x}_{B}-\mathbf{x}_{A}\right| / c$ being the travel time of the direct wave, propagating from $\mathbf{x}_{A}$ to $\mathbf{x}_{B}$. The Green's functions and source spectra appearing in (22) are all shown in Figure 2(a). Note 
a)

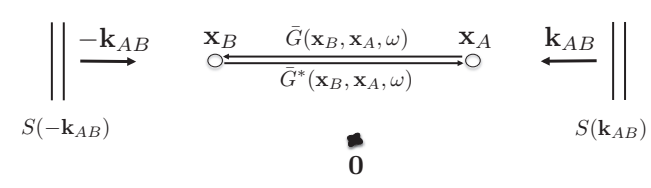

b)

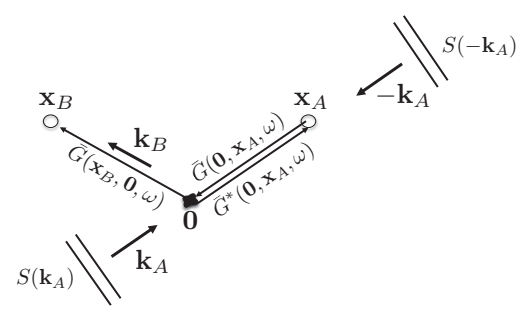

c)

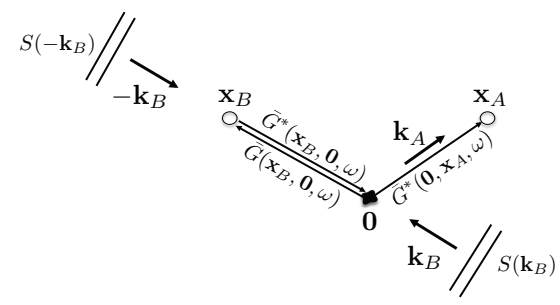

d)

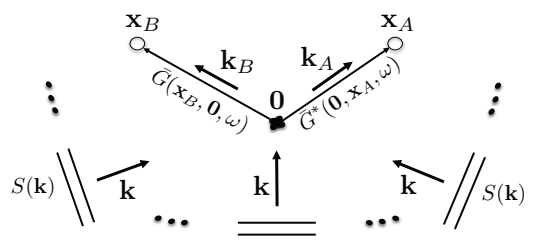

Figure 2. More detailed analysis of the dominating contributions to the correlation function $C\left(\mathbf{x}_{B}, \mathbf{x}_{A}, \omega\right)=$ $\mathcal{L}\left(\psi_{B}^{i}, \psi_{A}^{i}\right)+\mathcal{L}\left(\psi_{B}^{s}, \psi_{A}^{i}\right)+\mathcal{L}\left(\psi_{B}^{i}, \psi_{A}^{s}\right)+\mathcal{L}\left(\psi_{B}^{s}, \psi_{A}^{s}\right)$ (18). (a) The main contribution to $\mathcal{L}\left(\psi_{B}^{i}, \psi_{A}^{i}\right)$ (20) comes from waves propagating parallel to the line connecting the receivers at $\mathbf{x}_{A}$ and $\mathbf{x}_{B}$. (b) The main contribution to $\mathcal{L}\left(\psi_{B}^{s}, \psi_{A}^{i}\right)$ (26) comes from waves propagating parallel to the line connecting the receiver at $\mathbf{x}_{A}$ and the scatterer at the origin. (c) The main contribution to $\mathcal{L}\left(\psi_{B}^{i}, \psi_{A}^{s}\right)$ (33) comes from waves propagating parallel to the line connecting the receiver at $\mathbf{x}_{B}$ and the scatterer at the origin. (d) Waves propagating in all directions contribute to $\mathcal{L}\left(\psi_{B}^{s}, \psi_{A}^{s}\right)(37)$.

that $\bar{G}^{*}\left(\mathbf{x}_{B}, \mathbf{x}_{A}, \omega\right)$ is the Fourier transform of the time-reversed Green's function

$$
\bar{G}\left(\mathbf{x}_{B}, \mathbf{x}_{A},-t\right)=\frac{\delta\left(t+t_{\mathrm{dir}}\right)}{4 \pi\left|\mathbf{x}_{B}-\mathbf{x}_{A}\right|} .
$$

In Figure 2(a) this Green's function is pictured as back-propagating from $\mathbf{x}_{B}$ to $\mathbf{x}_{A}$.

For the second term in the right-hand side of (18) we obtain, using (2) and (3),

$$
\begin{aligned}
\mathcal{L}\left(\psi_{B}^{s}, \psi_{A}^{i}\right) & =\oint \psi^{s}\left(\mathbf{x}_{B}, \mathbf{k}\right)\left\{\psi^{i}\left(\mathbf{x}_{A}, \mathbf{k}\right)\right\}^{*} S(\mathbf{k}) \mathrm{d} \Omega_{k} \\
& =\frac{\exp \left(i k\left|\mathbf{x}_{B}\right|\right)}{\left|\mathbf{x}_{B}\right|} \oint \exp \left(-i \mathbf{k} \cdot \mathbf{x}_{A}\right) f\left(\mathbf{k}_{B}, \mathbf{k}\right) S(\mathbf{k}) \mathrm{d} \Omega_{k}
\end{aligned}
$$

with $\mathbf{k}_{B}=k \mathbf{x}_{B} /\left|\mathbf{x}_{B}\right|$. The stationary contributions occur for $\mathbf{k}$ parallel with $\mathbf{x}_{A}$, i.e., for 
$\mathbf{k}= \pm \mathbf{k}_{A}$ with $\mathbf{k}_{A}=k \mathbf{x}_{A} /\left|\mathbf{x}_{A}\right|$; see Figure 2(b). Hence (using again the result of Appendix A.1),

$$
\mathcal{L}\left(\psi_{B}^{s}, \psi_{A}^{i}\right)=\frac{8 \pi^{2}}{i k}\left[G^{s}\left(\mathbf{x}_{B}, \mathbf{x}_{A}, \omega\right) S\left(-\mathbf{k}_{A}\right)-\mathcal{M}_{1}\left(\mathbf{x}_{B}, \mathbf{x}_{A}, \omega\right) S\left(\mathbf{k}_{A}\right)\right],
$$

where $G^{s}\left(\mathbf{x}_{B}, \mathbf{x}_{A}, \omega\right)$ is the scattered part of the Green's function in the actual medium, i.e.,

$$
G^{s}\left(\mathbf{x}_{B}, \mathbf{x}_{A}, \omega\right)=4 \pi \bar{G}\left(\mathbf{x}_{B}, \mathbf{0}, \omega\right) f\left(\mathbf{k}_{B},-\mathbf{k}_{A}\right) \bar{G}\left(\mathbf{0}, \mathbf{x}_{A}, \omega\right),
$$

and where $\mathcal{M}_{1}\left(\mathbf{x}_{B}, \mathbf{x}_{A}, \omega\right)$ is defined as

$$
\mathcal{M}_{1}\left(\mathbf{x}_{B}, \mathbf{x}_{A}, \omega\right)=\mathcal{M}\left(\mathbf{x}_{B}, \mathbf{x}_{A}, \omega\right) f\left(\mathbf{k}_{B}, \mathbf{k}_{A}\right)
$$

with

$$
\mathcal{M}\left(\mathbf{x}_{B}, \mathbf{x}_{A}, \omega\right)=4 \pi \bar{G}\left(\mathbf{x}_{B}, \mathbf{0}, \omega\right) \bar{G}^{*}\left(\mathbf{0}, \mathbf{x}_{A}, \omega\right) .
$$

The forward propagating Green's functions appearing in the right-hand side of (28) are shown in Figure 2(b). Together with the scattering matrix $f\left(\mathbf{k}_{B},-\mathbf{k}_{A}\right)$ they constitute the scattered Green's function $G^{s}\left(\mathbf{x}_{B}, \mathbf{x}_{A}, \omega\right)$ in the actual medium. The inverse Fourier transform of $G^{s}\left(\mathbf{x}_{B}, \mathbf{x}_{A}, \omega\right)$ is

$$
\begin{aligned}
G^{s}\left(\mathbf{x}_{B}, \mathbf{x}_{A}, t\right) & =4 \pi \bar{G}\left(\mathbf{x}_{B}, \mathbf{0}, t\right) * f\left(\mathbf{n}_{B},-\mathbf{n}_{A}, t\right) * \bar{G}\left(\mathbf{0}, \mathbf{x}_{A}, t\right) \\
& =\frac{\delta\left(t-t_{\mathrm{sct}}\right)}{4 \pi\left|\mathbf{x}_{A}\right|\left|\mathbf{x}_{B}\right|} * f\left(\mathbf{n}_{B},-\mathbf{n}_{A}, t\right)=\frac{f\left(\mathbf{n}_{B},-\mathbf{n}_{A}, t-t_{\mathrm{sct}}\right)}{4 \pi\left|\mathbf{x}_{A}\right|\left|\mathbf{x}_{B}\right|},
\end{aligned}
$$

where $t_{\text {sct }}=\left|\mathbf{x}_{A}\right| / c+\left|\mathbf{x}_{B}\right| / c$ is the travel time along the path from $\mathbf{x}_{A}$, via the scatterer (which is centered around the origin), to $\mathbf{x}_{B}$. Hence, according to (31), $G^{s}\left(\mathbf{x}_{B}, \mathbf{x}_{A}, t\right)$ is proportional to the time-domain scattering matrix $f\left(\mathbf{n}_{B},-\mathbf{n}_{A}, t\right)$, delayed by $t_{\text {sct }}$. The function $\mathcal{M}_{1}\left(\mathbf{x}_{B}, \mathbf{x}_{A}, \omega\right)$, defined in (29) and (30), contains a forward and a backward propagating Green's function (Figure 2(b)) and has no physical meaning. The inverse Fourier transform of $\mathcal{M}_{1}\left(\mathbf{x}_{B}, \mathbf{x}_{A}, \omega\right)$ is

$$
\begin{aligned}
\mathcal{M}_{1}\left(\mathbf{x}_{B}, \mathbf{x}_{A}, t\right) & =4 \pi \bar{G}\left(\mathbf{x}_{B}, \mathbf{0}, t\right) * \bar{G}\left(\mathbf{0}, \mathbf{x}_{A},-t\right) * f\left(\mathbf{n}_{B}, \mathbf{n}_{A}, t\right) \\
& =\frac{\delta\left(t-t_{\mathrm{art}}\right)}{4 \pi\left|\mathbf{x}_{A}\right|\left|\mathbf{x}_{B}\right|} * f\left(\mathbf{n}_{B}, \mathbf{n}_{A}, t\right)=\frac{f\left(\mathbf{n}_{B}, \mathbf{n}_{A}, t-t_{\mathrm{art}}\right)}{4 \pi\left|\mathbf{x}_{A}\right|\left|\mathbf{x}_{B}\right|}
\end{aligned}
$$

with $t_{\text {art }}=\left|\mathbf{x}_{B}\right| / c-\left|\mathbf{x}_{A}\right| / c$. Hence, $\mathcal{M}_{1}\left(\mathbf{x}_{B}, \mathbf{x}_{A}, t\right)$ is an artifact, proportional to the scattering matrix $f\left(\mathbf{n}_{B}, \mathbf{n}_{A}, t\right)$, delayed by $t_{\text {art }}$.

In a similar way we find for the third term in the right-hand side of (18)

$$
\mathcal{L}\left(\psi_{B}^{i}, \psi_{A}^{s}\right)=\frac{8 \pi^{2}}{i k}\left[-\left\{G^{s}\left(\mathbf{x}_{B}, \mathbf{x}_{A}, \omega\right)\right\}^{*} S\left(-\mathbf{k}_{B}\right)+\mathcal{M}_{2}\left(\mathbf{x}_{B}, \mathbf{x}_{A}, \omega\right) S\left(\mathbf{k}_{B}\right)\right],
$$

see Figure 2(c), with

$$
\mathcal{M}_{2}\left(\mathbf{x}_{B}, \mathbf{x}_{A}, \omega\right)=\mathcal{M}\left(\mathbf{x}_{B}, \mathbf{x}_{A}, \omega\right) f^{*}\left(\mathbf{k}_{A}, \mathbf{k}_{B}\right) .
$$

Copyright (C) by SIAM. Unauthorized reproduction of this article is prohibited. 
The inverse Fourier transforms of $\left\{G^{s}\left(\mathbf{x}_{B}, \mathbf{x}_{A}, \omega\right)\right\}^{*}$ and $\mathcal{M}_{2}\left(\mathbf{x}_{B}, \mathbf{x}_{A}, \omega\right)$ are

$$
\begin{aligned}
G^{s}\left(\mathbf{x}_{B}, \mathbf{x}_{A},-t\right) & =\frac{f\left(\mathbf{n}_{B},-\mathbf{n}_{A},-t-t_{\mathrm{sct}}\right)}{4 \pi\left|\mathbf{x}_{A}\right|\left|\mathbf{x}_{B}\right|}, \\
\mathcal{M}_{2}\left(\mathbf{x}_{B}, \mathbf{x}_{A}, t\right) & =\frac{f\left(\mathbf{n}_{A}, \mathbf{n}_{B},-t+t_{\mathrm{art}}\right)}{4 \pi\left|\mathbf{x}_{A}\right|\left|\mathbf{x}_{B}\right|} .
\end{aligned}
$$

According to (35), $G^{s}\left(\mathbf{x}_{B}, \mathbf{x}_{A},-t\right)$ is proportional to the time-reversed scattering matrix, advanced by $t_{\text {sct }}$. According to $(36), \mathcal{M}_{2}\left(\mathbf{x}_{B}, \mathbf{x}_{A}, t\right)$ is proportional to the time-reversed scattering matrix, delayed by $t_{\text {art }}$.

Finally, for the last term in the right-hand side of (18) we find

$$
\begin{aligned}
\mathcal{L}\left(\psi_{B}^{s}, \psi_{A}^{s}\right) & =\oint \psi^{s}\left(\mathbf{x}_{B}, \mathbf{k}\right)\left\{\psi^{s}\left(\mathbf{x}_{A}, \mathbf{k}\right)\right\}^{*} S(\mathbf{k}) \mathrm{d} \Omega_{k} \\
& =4 \pi \mathcal{M}\left(\mathbf{x}_{B}, \mathbf{x}_{A}, \omega\right) \oint f\left(\mathbf{k}_{B}, \mathbf{k}\right) f^{*}\left(\mathbf{k}_{A}, \mathbf{k}\right) S(\mathbf{k}) \mathrm{d} \Omega_{k}
\end{aligned}
$$

see Figure 2(d). The integrand in (37) has no stationary contributions; hence, this expression is not further simplified. Like $\mathcal{M}_{1}\left(\mathbf{x}_{B}, \mathbf{x}_{A}, t\right)$ and $\mathcal{M}_{2}\left(\mathbf{x}_{B}, \mathbf{x}_{A}, t\right)$ in (32) and (36), (37) represents an artifact, proportional to a combination of scattering matrices, delayed by $t_{\text {art }}$.

Combining all terms gives, in the frequency domain,

$$
\begin{aligned}
& C\left(\mathbf{x}_{B}, \mathbf{x}_{A}, \omega\right)=\frac{8 \pi^{2}}{i k}[ \bar{G}\left(\mathbf{x}_{B}, \mathbf{x}_{A}, \omega\right) S\left(\mathbf{k}_{A B}\right)-\bar{G}^{*}\left(\mathbf{x}_{B}, \mathbf{x}_{A}, \omega\right) S\left(-\mathbf{k}_{A B}\right) \\
&\left.+G^{s}\left(\mathbf{x}_{B}, \mathbf{x}_{A}, \omega\right) S\left(-\mathbf{k}_{A}\right)-\left\{G^{s}\left(\mathbf{x}_{B}, \mathbf{x}_{A}, \omega\right)\right\}^{*} S\left(-\mathbf{k}_{B}\right)\right] \\
&-\frac{16 \pi^{2}}{k} \mathcal{M}\left(\mathbf{x}_{B}, \mathbf{x}_{A}, \omega\right)\left[\frac{1}{2 i}\left\{f\left(\mathbf{k}_{B}, \mathbf{k}_{A}\right) S\left(\mathbf{k}_{A}\right)-f^{*}\left(\mathbf{k}_{A}, \mathbf{k}_{B}\right) S\left(\mathbf{k}_{B}\right)\right\}\right. \\
&\left.-\frac{k}{4 \pi} \oint f\left(\mathbf{k}_{B}, \mathbf{k}\right) f^{*}\left(\mathbf{k}_{A}, \mathbf{k}\right) S(\mathbf{k}) \mathrm{d} \Omega_{k}\right] .
\end{aligned}
$$

For the special case of a diffuse field (i.e., an equipartitioned noise field) we may replace $S\left(\mathbf{k}_{A B}\right), S\left(-\mathbf{k}_{A B}\right)$, etc., by one-and-the-same spectrum $S_{0}(\omega)$. For this situation the last term in (38) vanishes on account of the optical theorem (6) and the symmetry relation (5) for the scattering matrix $[32,36,37]$. This leaves

$$
C\left(\mathbf{x}_{B}, \mathbf{x}_{A}, \omega\right)=\frac{8 \pi^{2}}{i k}\left[G\left(\mathbf{x}_{B}, \mathbf{x}_{A}, \omega\right)-G^{*}\left(\mathbf{x}_{B}, \mathbf{x}_{A}, \omega\right)\right] S_{0}(\omega)
$$

where $G\left(\mathbf{x}_{B}, \mathbf{x}_{A}, \omega\right)$ is the total Green's function in the actual medium, i.e.,

$$
G\left(\mathbf{x}_{B}, \mathbf{x}_{A}, \omega\right)=\bar{G}\left(\mathbf{x}_{B}, \mathbf{x}_{A}, \omega\right)+G^{s}\left(\mathbf{x}_{B}, \mathbf{x}_{A}, \omega\right) .
$$

Equation (39) formulates the well-known relation between the correlation function and the Green's function for the situation that the noise field is diffuse $[1,3,4,5,6]$. Equation (38) is the generalization of this relation for directional noise. The different contributions to the

Copyright (C) by SIAM. Unauthorized reproduction of this article is prohibited. 
Green's functions in the right-hand side of (38) are weighted by different power spectra, or, in the time domain, convolved with different autocorrelation functions. Moreover, the right-hand side of (38) contains a nonvanishing term proportional to $\mathcal{M}\left(\mathbf{x}_{B}, \mathbf{x}_{A}, \omega\right)$, corresponding to a superposition of artifacts around $t=t_{\text {art }}$ in the time domain. In sections 4 and 5 we use (38) together with (28) as the basis for retrieving the directional scattering matrix from directional noise.

3.2. 2D situation. For the $2 \mathrm{D}$ situation, we obtain instead of (38) (using Appendix A.2)

$$
\begin{aligned}
& C\left(\mathbf{x}_{B}, \mathbf{x}_{A}, \omega\right)=-4 i \mu \pi[ \bar{G}\left(\mathbf{x}_{B}, \mathbf{x}_{A}, \omega\right) S\left(\mathbf{k}_{A B}\right)-\bar{G}^{*}\left(\mathbf{x}_{B}, \mathbf{x}_{A}, \omega\right) S\left(-\mathbf{k}_{A B}\right) \\
&\left.+G^{s}\left(\mathbf{x}_{B}, \mathbf{x}_{A}, \omega\right) S\left(-\mathbf{k}_{A}\right)-\left\{G^{s}\left(\mathbf{x}_{B}, \mathbf{x}_{A}, \omega\right)\right\}^{*} S\left(-\mathbf{k}_{B}\right)\right] \\
&-8 \mu \pi \mathcal{M}\left(\mathbf{x}_{B}, \mathbf{x}_{A}, \omega\right) {\left[\frac{1}{2 i}\left\{f\left(\mathbf{k}_{B}, \mathbf{k}_{A}\right) S\left(\mathbf{k}_{A}\right)-f^{*}\left(\mathbf{k}_{A}, \mathbf{k}_{B}\right) S\left(\mathbf{k}_{B}\right)\right\}\right.} \\
&\left.-\frac{\mu}{\sqrt{8 \pi}} \oint f\left(\mathbf{k}_{B}, \mathbf{k}\right) f^{*}\left(\mathbf{k}_{A}, \mathbf{k}\right) S(\mathbf{k}) \mathrm{d} \Omega_{k}\right]
\end{aligned}
$$

with

$$
\begin{aligned}
\bar{G}\left(\mathbf{x}_{B}, \mathbf{x}_{A}, \omega\right) & =\frac{\exp \left(i k\left|\mathbf{x}_{B}-\mathbf{x}_{A}\right|+i \mu \pi / 4\right)}{\sqrt{8 \pi|k|\left|\mathbf{x}_{B}-\mathbf{x}_{A}\right|}} \\
G^{s}\left(\mathbf{x}_{B}, \mathbf{x}_{A}, \omega\right) & =\sqrt{8 \pi} \bar{G}\left(\mathbf{x}_{B}, \mathbf{0}, \omega\right) f\left(\mathbf{k}_{B},-\mathbf{k}_{A}\right) \bar{G}\left(\mathbf{0}, \mathbf{x}_{A}, \omega\right),
\end{aligned}
$$

and

$$
\mathcal{M}\left(\mathbf{x}_{B}, \mathbf{x}_{A}, \omega\right)=\sqrt{8 \pi} \bar{G}\left(\mathbf{x}_{B}, \mathbf{0}, \omega\right) \bar{G}^{*}\left(\mathbf{0}, \mathbf{x}_{A}, \omega\right) .
$$

\section{Properties of the correlation function.}

4.1. 3D situation. In (15) we defined the correlation function $C\left(\mathbf{x}_{B}, \mathbf{x}_{A}, t\right)$ as the crosscorrelation of responses at two receivers $\mathbf{x}_{A}$ and $\mathbf{x}_{B}$. Equation (38) formulates the relation between the Fourier transform of the correlation function and the Green's function. Summarizing the time-domain expressions of section 3.1, the correlation function consists of

- two events at $\pm t_{\text {dir }}$, where $t_{\text {dir }}=\left|\mathbf{x}_{B}-\mathbf{x}_{A}\right| / c$ is the travel time of the direct wave between $\mathbf{x}_{A}$ and $\mathbf{x}_{B}$,

- one time-domain scattering matrix, delayed by $t_{\mathrm{sct}}$, and one time-reversed scattering matrix, advanced by $t_{\mathrm{sct}}$, where $t_{\mathrm{sct}}=\left|\mathbf{x}_{A}\right| / c+\left|\mathbf{x}_{B}\right| / c$ is the travel time along the path from $\mathbf{x}_{A}$, via the scatterer, to $\mathbf{x}_{B}$,

- a superposition of artifacts, delayed by $t_{\text {art }}$, with $t_{\text {art }}=\left|\mathbf{x}_{B}\right| / c-\left|\mathbf{x}_{A}\right| / c$; these artifacts only cancel when the noise is equipartitioned.

Each of these contributions is convolved with a specific noise autocorrelation function and is time-integrated (due to the factors $1 / i k$ ). Note that $\left|t_{\text {art }}\right| \leq t_{\text {dir }} \leq t_{\text {sct }}$. When $\mathbf{x}_{A}$ and $\mathbf{x}_{B}$ lie at opposite sides on a straight line through the origin, $t_{\text {dir }}=t_{\text {sct }}$, meaning that the direct wave contributions interfere with the forward scattered contributions. On the other hand, when $\mathbf{x}_{A}$ and $\mathbf{x}_{B}$ lie both at the same side on a straight line through the origin, $t_{\text {dir }}=\left|t_{\text {art }}\right|$,

Copyright (C) by SIAM. Unauthorized reproduction of this article is prohibited. 
meaning that the artifact interferes with one of the direct wave contributions (or with both when $\left.t_{\text {art }}=0\right)$. Finally, for those situations where $t_{\text {dir }}+d t / 2<t_{\text {sct }}-d t / 2$, where $d t$ is a measure for the duration of the autocorrelation of the noise, the causal and acausal scattered events can be selected from the total correlation function by time-windowing. This condition is fulfilled when $\mathbf{x}_{A}$ and $\mathbf{x}_{B}$ do not lie approximately at opposite sides on a straight line through the origin. We define windowed correlation functions as

$$
\begin{aligned}
& C^{s}\left(\mathbf{x}_{B}, \mathbf{x}_{A}, t\right)=H\left(t-t_{\mathrm{sct}}+d t / 2\right) C\left(\mathbf{x}_{B}, \mathbf{x}_{A}, t\right), \\
& C_{a}^{s}\left(\mathbf{x}_{B}, \mathbf{x}_{A}, t\right)=H\left(-t-t_{\mathrm{sct}}+d t / 2\right) C\left(\mathbf{x}_{B}, \mathbf{x}_{A}, t\right),
\end{aligned}
$$

where superscript $s$ stands for "scattered," subscript a stands for "acausal," and $H(t)$ is the Heaviside step function. The Fourier transforms of these windowed correlation functions correspond to the scattered Green's functions at the right-hand side of (38), as follows:

$$
\begin{aligned}
C^{s}\left(\mathbf{x}_{B}, \mathbf{x}_{A}, \omega\right) & =\frac{8 \pi^{2}}{i k} G^{s}\left(\mathbf{x}_{B}, \mathbf{x}_{A}, \omega\right) S\left(-\mathbf{k}_{A}\right), \\
\left\{C_{a}^{s}\left(\mathbf{x}_{B}, \mathbf{x}_{A}, \omega\right)\right\}^{*} & =\frac{8 \pi^{2}}{i k} G^{s}\left(\mathbf{x}_{B}, \mathbf{x}_{A}, \omega\right) S\left(-\mathbf{k}_{B}\right) .
\end{aligned}
$$

From the discussion above it follows that this separation is possible when $\mathbf{x}_{A}$ and $\mathbf{x}_{B}$ do not lie approximately at opposite sides on a straight line through the origin, i.e., when $\mathbf{k}_{B}$ is not approximately equal to $-\mathbf{k}_{A}$. Hence, except for (approximate) forward scattering, we find

$$
\frac{\left\{C_{a}^{s}\left(\mathbf{x}_{B}, \mathbf{x}_{A}, \omega\right)\right\}^{*}}{C^{s}\left(\mathbf{x}_{B}, \mathbf{x}_{A}, \omega\right)}=\frac{S\left(-\mathbf{k}_{B}\right)}{S\left(-\mathbf{k}_{A}\right)} .
$$

4.2. 2D situation. For the $2 \mathrm{D}$ situation, (47) and (48) need to be replaced by

$$
\begin{aligned}
C^{s}\left(\mathbf{x}_{B}, \mathbf{x}_{A}, \omega\right) & =-4 i \mu \pi G^{s}\left(\mathbf{x}_{B}, \mathbf{x}_{A}, \omega\right) S\left(-\mathbf{k}_{A}\right), \\
\left\{C_{a}^{s}\left(\mathbf{x}_{B}, \mathbf{x}_{A}, \omega\right)\right\}^{*} & =-4 i \mu \pi G^{s}\left(\mathbf{x}_{B}, \mathbf{x}_{A}, \omega\right) S\left(-\mathbf{k}_{B}\right) .
\end{aligned}
$$

Equation (49) remains unchanged. In the next section we illustrate with a numerical example how this can be used to estimate (apart from a scaling factor) the directional power spectrum of the noise field and, subsequently, the scattering matrix.

5. Retrieving the noise directivity and the directional scattering matrix from the correlation function. Figure 3 shows a scattering object in a homogeneous embedding medium. The propagation velocity of the embedding medium is $2000 \mathrm{~m} / \mathrm{s}$ and the mass density 1000 $\mathrm{kg} / \mathrm{m}^{3}$. The scattering object has a velocity of $6000 \mathrm{~m} / \mathrm{s}$ and a mass density of $5000 \mathrm{~kg} / \mathrm{m}^{3}$. A circular array of 180 receivers is placed around the scatterer; see Figure 4. The radius $r$ of the array is $50 \mathrm{~mm}$. The configuration is illuminated by a directional noise field, consisting of 1440 mutually uncorrelated plane waves (simulated by distant monopole point sources at a circular array with a radius of $125 \mathrm{~mm}$ ), of which the average amplitude varies with the illumination angle, according to

$$
A(\alpha)=1+0.5 \cos \left(\alpha+60^{\circ}\right)
$$

Copyright (c) by SIAM. Unauthorized reproduction of this article is prohibited. 


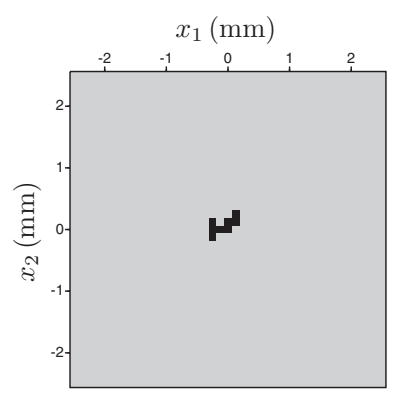

Figure 3. Scattering object used in the numerical experiment.

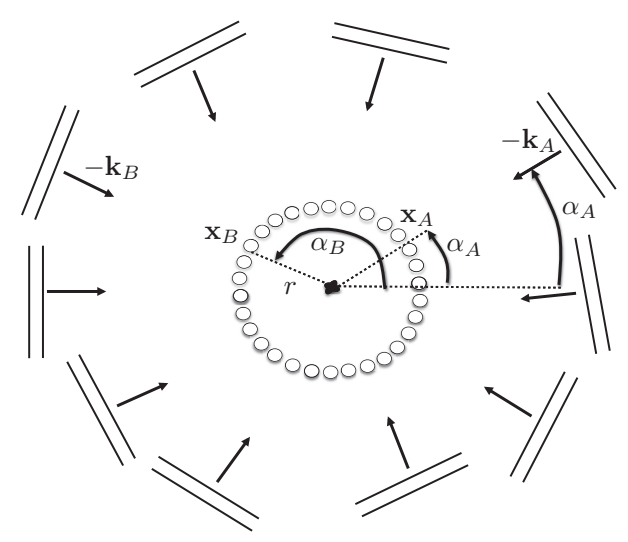

Figure 4. Acquisition configuration for the numerical experiment. The radius of the receiver array is $50 \mathrm{~mm}$.

The angle-dependent autocorrelation of the noise is $A^{2}(\alpha) S_{0}(t)$ with $S_{0}(t)$ defined as

$$
S_{0}(t)=\left(1-\omega_{0}^{2} t^{2} / 2\right) \exp \left(-\omega_{0}^{2} t^{2} / 4\right)
$$

with $\omega_{0} / 2 \pi=800 \mathrm{kHz}$. The corresponding power spectrum is $A^{2}(\alpha) S_{0}(\omega)$, with

$$
S_{0}(\omega)=4 \sqrt{\pi} \frac{\omega^{2}}{\omega_{0}^{3}} \exp \left(-\omega^{2} / \omega_{0}^{2}\right) .
$$

The receivers sample the noise field (acoustic pressure) with a sampling interval of $0.05 \mu \mathrm{s}$ and the total duration of the noise registrations is approximately 10 minutes. In the following the receiver coordinates $\mathbf{x}_{A}$ and $\mathbf{x}_{B}$, both at distance $r$ from the origin, will be represented by angles $\alpha_{A}$ and $\alpha_{B}$, respectively (see Figure 4 ). The crosscorrelation, defined in (15), reads $C\left(\alpha_{B}, \alpha_{A}, r, t\right)$ in these polar coordinates. We evaluate the crosscorrelation by dividing the noise registrations in blocks of 65,536 samples, computing the crosscorrelation per block via the frequency domain and averaging the results over the blocks. Figure 5 shows the correlation function $C\left(\alpha_{B}, \alpha_{A}, r, t\right)$ for fixed $\alpha_{A}=0^{\circ}$ and variable $\alpha_{B}$ and $t$. (Figures 5(a) and 5(b) represent this correlation function in gray-level and wiggle display, respectively.)

We clearly recognize the events discussed in section 4.1. The two curved events represent the retrieved direct waves at $t= \pm t_{\text {dir }}$ (weighted by the directivity of the noise), the events at 

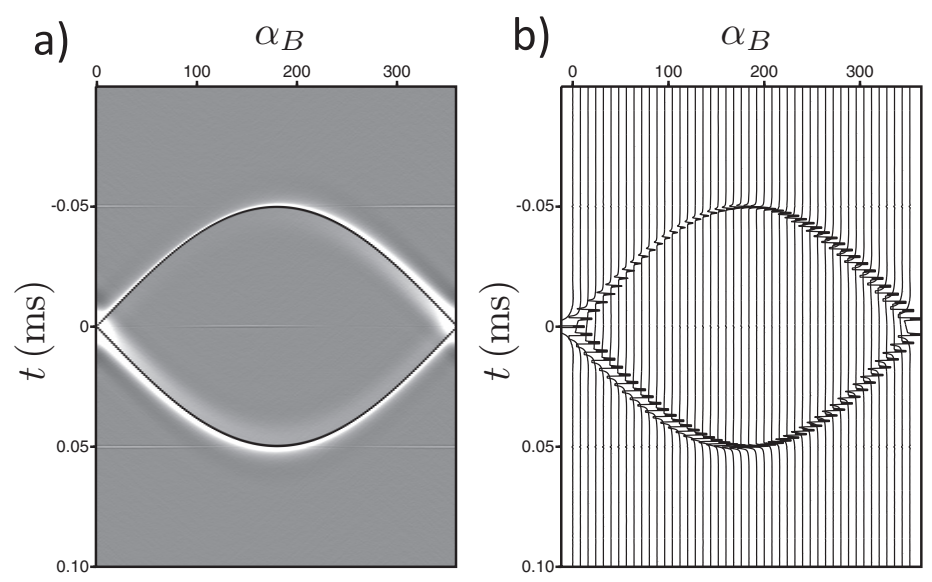

Figure 5. Correlation function $C\left(\alpha_{B}, \alpha_{A}, r, t\right)$ for fixed $\alpha_{A}=0^{\circ}$ and variable $\alpha_{B}$ and $t$. (a) Gray-level display. (b) Wiggle display.

$t= \pm t_{\mathrm{sct}}= \pm 2 r / c= \pm 0.05 \mathrm{~ms}$ are the retrieved scattered waves, and the event at $t=t_{\mathrm{art}}=0$ $\mathrm{ms}$ is the nonvanishing artifact, resulting from the directional behavior of the noise field.

Note that for this example the direct waves could be used to estimate the directional properties of the noise field, which could subsequently be used to estimate the directional scattering matrix from the scattered waves. However, according to (41) the direct and scattered waves are weighted with unrelated components of the power spectrum of the directional noise field $\left[S\left(\mathbf{k}_{A B}\right)\right.$ versus $S\left(-\mathbf{k}_{A}\right)$, etc.]. Although one could correct for this in the case of a full receiver aperture, in more realistic situations this could bias the estimated scattering matrix. In the case of a limited receiver aperture, as discussed at the end of this section, it is even impossible to estimate the required directional properties of the noise from the direct waves. Therefore we prefer to derive the directional properties of the noise field and of the scattering matrix both from the scattered waves.

The scattered waves can be selected from Figure 5 by time-windowing. Figure 6(a) shows the causal correlation function $C^{s}\left(\alpha_{B}, \alpha_{A}=0, r, t\right)((45)$ in polar coordinates) and Figure 6(b) the time-reversal of the acausal correlation function, i.e., $C_{a}^{s}\left(\alpha_{B}, \alpha_{A}=0, r,-t\right)$ (46). Note that these figures clearly show the coda of the time-dependent scattering matrix $f\left(\alpha_{B}, 0, t\right)$. The gap in both figures is a result of the interference of the direct and the forward scattered waves in Figure 5 around $180^{\circ}$. We repeat the correlation and windowing procedure for other values of $\alpha_{A}$ and take the Fourier transform of $C^{s}\left(\alpha_{B}, \alpha_{A}, r, t\right)$ and $C_{a}^{s}\left(\alpha_{B}, \alpha_{A}, r,-t\right)$, giving $C^{s}\left(\alpha_{B}, \alpha_{A}, r, \omega\right)$ and $\left\{C_{a}^{s}\left(\alpha_{B}, \alpha_{A}, r, \omega\right)\right\}^{*}$, respectively. Figures 7 (a) and 7(b) show the modulus of these functions for frequency $\omega / 2 \pi=732 \mathrm{kHz}$. According to (50) and (51), both figures represent the scattered part of the Green's function, weighted by the power spectra $S\left(-\mathbf{k}_{A}\right)=A^{2}\left(\alpha_{A}\right) S_{0}(\omega)$ and $S\left(-\mathbf{k}_{B}\right)=A^{2}\left(\alpha_{B}\right) S_{0}(\omega)$, respectively. (Here we relate the vectors $-\mathbf{k}_{A}$ and $-\mathbf{k}_{B}$ to $\alpha_{A}$ and $\alpha_{B}$, respectively, see Figure 4.) The blue diagonal bands represent the gaps caused by the interference of the direct and the forward scattered waves. According to (49), the ratio of Figures 7(b) and 7(a), i.e., $R\left(\alpha_{B}, \alpha_{A}, \omega\right)=\left\{C_{a}^{s}\left(\alpha_{B}, \alpha_{A}, r, \omega\right)\right\}^{*} / C^{s}\left(\alpha_{B}, \alpha_{A}, r, \omega\right)$, gives (except in the gaps) the ratio of 

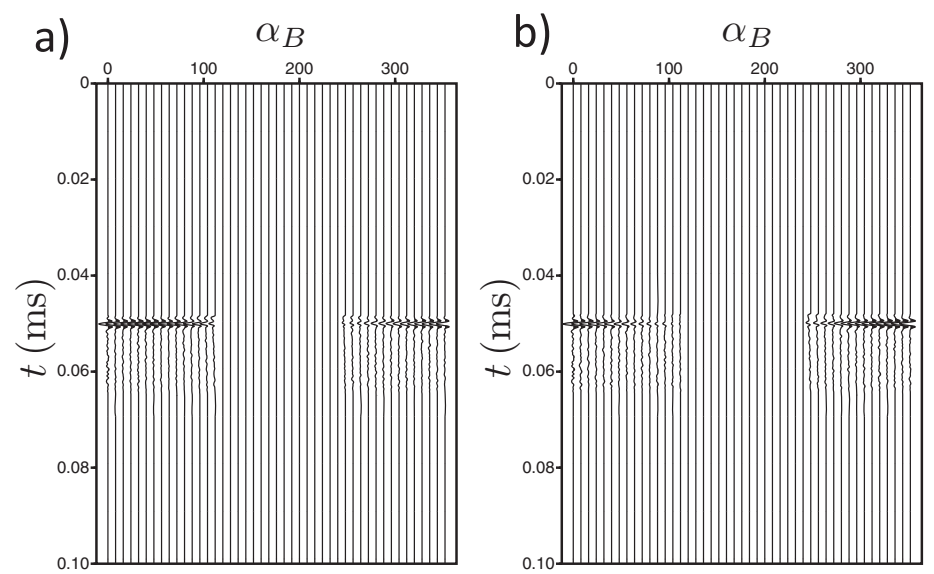

Figure 6. (a) Causal correlation function $C^{s}\left(\alpha_{B}, \alpha_{A}=0, r, t\right)$. (b) Time-reversal of the acausal correlation function, i.e., $C_{a}^{s}\left(\alpha_{B}, \alpha_{A}=0, r,-t\right)$.
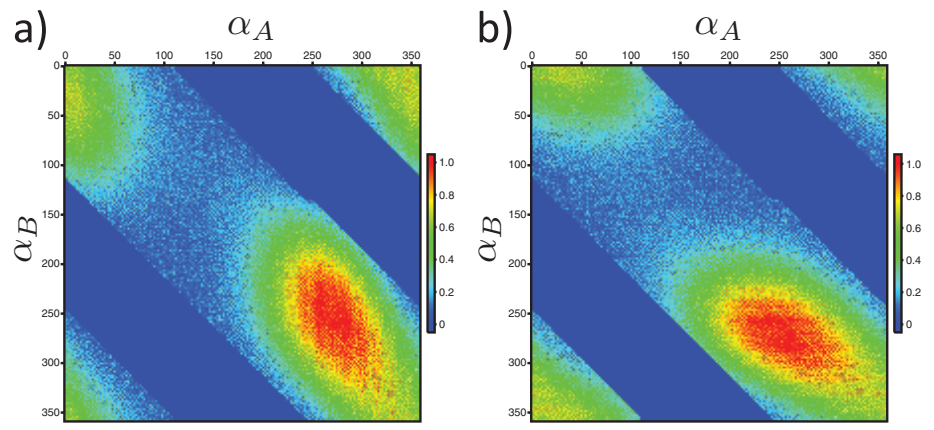

Figure 7. One frequency slice of the modulus of (a) $C^{s}\left(\alpha_{B}, \alpha_{A}, r, \omega\right)$ and (b) $\left\{C_{a}^{s}\left(\alpha_{B}, \alpha_{A}, r, \omega\right)\right\}^{*}$. Here and in subsequent figures the color scales represent relative amplitudes.

the power spectra $S\left(-\mathbf{k}_{B}\right) / S\left(-\mathbf{k}_{A}\right)=A^{2}\left(\alpha_{B}\right) / A^{2}\left(\alpha_{A}\right)$, hence

$$
R\left(\alpha_{B}, \alpha_{A}, \omega\right)=\frac{\left\{C_{a}^{s}\left(\alpha_{B}, \alpha_{A}, r, \omega\right)\right\}^{*}}{C^{s}\left(\alpha_{B}, \alpha_{A}, r, \omega\right)}=\frac{A^{2}\left(\alpha_{B}\right)}{A^{2}\left(\alpha_{A}\right)} .
$$

We define a matrix $\mathbf{R}$ containing the frequency-averaged ratio $R\left(\alpha_{B}, \alpha_{A}\right)$ (where outliers are exluded from the averaging process). Moreover, we define vectors $\mathbf{s}$ and $\mathbf{q}$, containing $A^{2}(\alpha)$ and $1 / A^{2}(\alpha)$, respectively. With these definitions, (55) becomes

$$
\mathbf{R}=\mathbf{s q}^{t},
$$

where superscript $t$ denotes transposition. Because vectors $\mathbf{s}$ and $\mathbf{q}$ are mutually related, they can be estimated from (56), apart from an undetermined scaling factor. Note that $\mathbf{R} \mathbf{s}=\mathbf{s q}^{t} \mathbf{s}=\mathbf{s} N_{r}$, where $N_{r}=\mathbf{q}^{t} \mathbf{s}$ equals the number of receivers in the receiver array (here $\left.N_{r}=180\right)$. Hence, $\mathbf{s}=N_{r}^{-1} \mathbf{R s}$. This suggests the following iterative scheme to resolve $\mathbf{s}$ from R:

$$
\mathbf{s}_{i+1}=\frac{1}{\gamma N_{r}} \mathbf{R s}_{i}, \quad \mathbf{s}_{1}=\left(\begin{array}{llll}
1 & 1 & \cdots & 1
\end{array}\right)^{t},
$$

Copyright ( $)$ by SIAM. Unauthorized reproduction of this article is prohibited. 


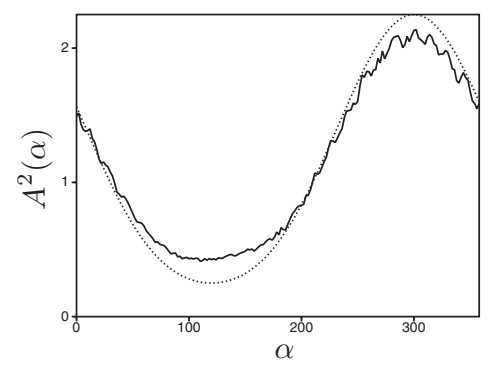

Figure 8. Estimated angle-dependent behavior of the power spectrum of the noise (solid), compared with the modeled version (dotted).
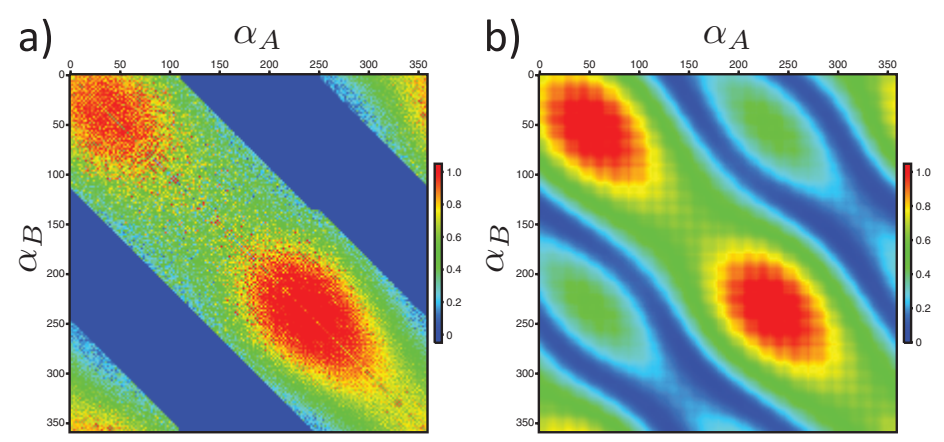

Figure 9. One frequency slice of the modulus of $G^{s}\left(\alpha_{B}, \alpha_{A}, r, \omega\right) S_{0}(\omega)$ or, equivalently, of the scattering matrix $f\left(\alpha_{B}, \alpha_{A}, \omega\right)$. (a) Estimated from directional noise. (b) Directly modeled version.

where $\gamma$ is a factor between zero and one to compensate for the gaps in $\mathbf{R}$. We choose $\gamma=0.61$, being the fraction of receivers for which the direct and scattered waves do not interfere. Applying the iterative scheme of (57) yields an estimate of $\mathbf{s}$ and, hence, of the angle-dependent weighting function $A^{2}(\alpha)$. The estimated function $A^{2}(\alpha)$, obtained after 18 iterations, is shown in Figure 8 together with the modeled version of this function, obtained directly from (52). Here the estimated curve has been scaled by a constant factor for ease of comparison, but this scaling factor is not used in the following. Note that the variation with angle is somewhat underestimated, mainly because amplitudes are averaged over a Fresnel zone.

Next, $G^{s}\left(\alpha_{B}, \alpha_{A}, r, \omega\right) S_{0}(\omega)$ can be obtained by inverting (50) and (51), i.e., by removing the angle-dependent weighting functions $A^{2}\left(\alpha_{A}\right)$ and $A^{2}\left(\alpha_{B}\right)$ from Figures $7(\mathrm{a})$ and $7(\mathrm{~b})$, respectively. Both inversions should converge to the same result, but the signal-to-noise ratio improves by averaging the two results, according to

$$
G^{s}\left(\alpha_{B}, \alpha_{A}, r, \omega\right) S_{0}(\omega)=\frac{1}{2}\left[\frac{C^{s}\left(\alpha_{B}, \alpha_{A}, r, \omega\right)}{-4 i \mu \pi A^{2}\left(\alpha_{A}\right)}+\frac{\left\{C_{a}^{s}\left(\alpha_{B}, \alpha_{A}, r, \omega\right)\right\}^{*}}{-4 i \mu \pi A^{2}\left(\alpha_{B}\right)}\right] .
$$

The modulus of this average is shown in Figure 9 (a) for $\omega / 2 \pi=732 \mathrm{kHz}$, where it is compared with the directly modeled scattered Green's function for the same frequency (Figure 9(b)). In both figures the color scale is adapted to the maximum of the plotted function, hence, the effect of the undetermined scaling factor is not visible. According to (42) and (43), 
a)

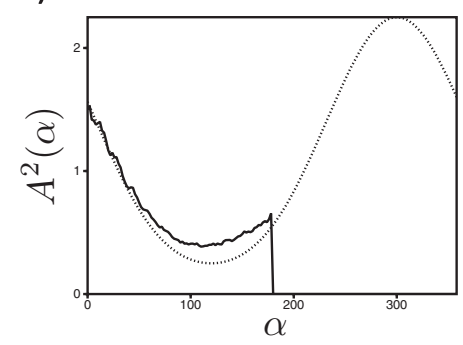

b)

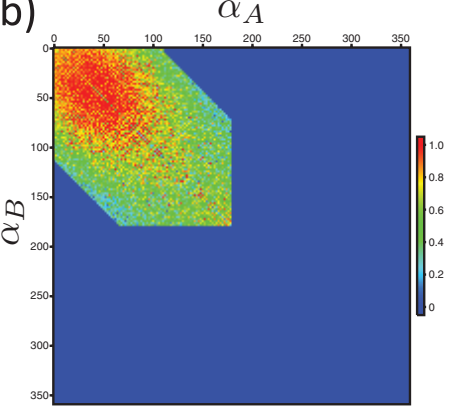

Figure 10. As in Figures 8 and 9 but for a limited receiver aperture from $0^{\circ}$ to $180^{\circ}$. (a) Estimated power spectrum of the noise. (b) Estimated scattering matrix.

a)

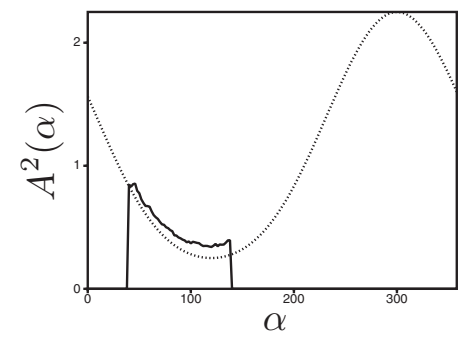

b)

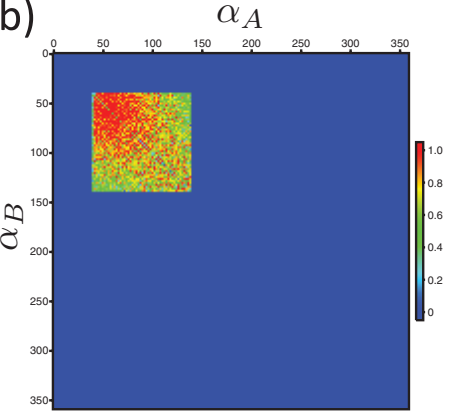

Figure 11. As in Figure 10 but for a limited receiver aperture from $40^{\circ}$ to $140^{\circ}$.

$G^{s}\left(\alpha_{B}, \alpha_{A}, r, \omega\right) S_{0}(\omega)$ is proportional to the angle-dependent scattering matrix $f\left(\alpha_{B}, \alpha_{A}, \omega\right)$ (via a frequency-dependent factor). Inverting this gives

$$
f\left(\alpha_{B}, \alpha_{A}, \omega\right)=-i \sqrt{8 \pi} k r \exp (-2 i k r) G^{s}\left(\alpha_{B}, \alpha_{A}, r, \omega\right) .
$$

Hence, Figures 9(a) and 9(b) can alternatively be interpreted as the estimated and modeled versions of the modulus of the angle-dependent scattering matrix. (Because the color scale is adapted to the maximum, the factor in (59) has no effect on the display.) We may conclude from Figure 9 that the scattering matrix has been retrieved reasonably accurately from the directional noise, except in the gaps caused by the interference with the forward scattered waves. The result in Figure 9(a) is a significantly better estimate of the scattering matrix in Figure 9 (b) than the uncorrected causal and acausal correlation functions in Figures 7(a) and $7(\mathrm{~b})$.

In many practical situations only a partial receiver aperture will be available. We simulate this by repeating the procedure described above, using only a subset of the 180 receivers of the circular array of Figure 4. Using the 90 receivers between $0^{\circ}$ and $180^{\circ}$ we recover the angle-dependent power spectrum $A^{2}(\alpha)$ of the noise (Figure 10(a)) and the scattering matrix $f\left(\alpha_{B}, \alpha_{A}, \omega\right)$ (Figure 10(b)) for this limited angle range with the same accuracy as obtained with the full receiver aperture (Figures 8 and 9(a)). By further reducing the number of receivers to 50 (between $40^{\circ}$ and $140^{\circ}$ ), we retrieve the power spectrum (Figure 11(a)) and

Copyright (c) by SIAM. Unauthorized reproduction of this article is prohibited. 
scattering matrix (Figure 11(b)), again with the same accuracy as before but of course for a limited angle range.

The latter two examples show that the proposed method does not require a complete circular receiver array and that with a limited receiver aperture the noise directivity properties and the scattering matrix can be accurately retrieved within the angle range covered by the receiver aperture.

In all examples the receiver array was regular and therefore the relatively simple iterative scheme (57) worked very well. For incomplete receiver arrays or corrupted data, matrix $\mathbf{R}$ needs to be reconstructed as well as possible prior to applying the iterative scheme. One of the reviewers pointed out that the principal component pursuit method may give very good results $[38,39,40,41]$.

6. Concluding remarks. The correlation of noise measurements at two receivers converges to the impulse response between these receivers, assuming the noise field is diffuse. In many practical situations the noise field is not purely diffuse. For the situation of a directional scatterer in a homogeneous embedding medium, illuminated by a directional noise field, the correlation function is proportional to the product of the directionalities of the scatterer and of the noise field. Using a relation between the causal and the acausal part of the correlation function, we have derived a method to resolve both directionalities, apart from an undetermined scaling factor, from the correlation function. For the situation of a circular receiver array around the scattering object we have shown that the noise directivity properties can be retrieved for all angles, whereas the scattering matrix can be retrieved for all combinations of incidence and scattering angles, except in a region around forward scattering because of interference with the direct wave. For the situation of a limited receiver aperture, the noise directivity properties and the scattering matrix can be retrieved within the angle range covered by the receivers (except, again, of course, in the region around forward scattering).

The proposed method has the potential to retrieve the scattering matrix of a compact scattering object from ambient noise in a much more accurate way than the straightforward crosscorrelation method.

\section{Appendix A. Stationary-phase analysis.}

A.1. 3D situation. We analyze the integral

$$
\mathcal{I}=\oint \exp \left(i \mathbf{k} \cdot \mathbf{x}_{0}\right) u(\mathbf{k}) \mathrm{d} \Omega_{k}
$$

with the method of stationary phase $[42,43]$. We express $\mathbf{k}$ and $\mathbf{x}_{0}$ in spherical coordinates with angles $\alpha$ and $\beta$ relative to $\mathbf{x}_{0}$, as

$$
\mathbf{k}=k\left(\begin{array}{c}
\cos \alpha \sin \beta \\
\sin \alpha \sin \beta \\
\cos \beta
\end{array}\right), \quad \mathbf{x}_{0}=\left|\mathbf{x}_{0}\right|\left(\begin{array}{l}
1 \\
0 \\
0
\end{array}\right) \text {. }
$$

Hence,

$$
\mathcal{I}=\int_{0}^{2 \pi} \mathrm{d} \alpha \int_{0}^{\pi} \exp \left(i k\left|\mathbf{x}_{0}\right| \cos \alpha \sin \beta\right) u(k, \alpha, \beta) \sin \beta \mathrm{d} \beta
$$

Copyright (c) by SIAM. Unauthorized reproduction of this article is prohibited. 
First we consider the $\beta$-integral. For the argument of the exponential function we write $i k \psi(\beta)$, where the phase function $\psi(\beta)$ is defined as $\psi(\beta)=\left|\mathbf{x}_{0}\right| \cos \alpha \sin \beta$. Differentiation with respect to $\beta$ gives $\psi^{\prime}(\beta)=\left|\mathbf{x}_{0}\right| \cos \alpha \cos \beta$ and $\psi^{\prime \prime}(\beta)=-\left|\mathbf{x}_{0}\right| \cos \alpha \sin \beta$. Note that $\psi^{\prime}(\pi / 2)=0$, hence $\beta_{0}=\pi / 2$ is the stationary point of the $\beta$-integral. For large $|k|$ we thus obtain

$$
\begin{aligned}
\mathcal{I} & \approx \int_{0}^{2 \pi} \sqrt{\frac{2 \pi}{\left|k \psi^{\prime \prime}\left(\beta_{0}\right)\right|}} \exp \left\{i\left(k \psi\left(\beta_{0}\right)+\nu \pi / 4\right)\right\} u\left(k, \alpha, \beta_{0}\right) \sin \beta_{0} \mathrm{~d} \alpha \\
& =\int_{0}^{2 \pi} \sqrt{\frac{2 \pi}{|k \cos \alpha|\left|\mathbf{x}_{0}\right|}} \exp \left\{i\left(k\left|\mathbf{x}_{0}\right| \cos \alpha+\nu \pi / 4\right)\right\} u(k, \alpha, \pi / 2) \mathrm{d} \alpha
\end{aligned}
$$

with $\nu=\operatorname{sign}\left(k \psi^{\prime \prime}\left(\beta_{0}\right)\right)=\operatorname{sign}(-k \cos \alpha)$. Next we consider the $\alpha$-integral. We define a phase function $\phi(\alpha)$ as $\phi(\alpha)=\left|\mathbf{x}_{0}\right| \cos \alpha$. Differentiation with respect to $\alpha$ gives $\phi^{\prime}(\alpha)=-\left|\mathbf{x}_{0}\right| \sin \alpha$ and $\phi^{\prime \prime}(\alpha)=-\left|\mathbf{x}_{0}\right| \cos \alpha$. Note that $\phi^{\prime}(0)=\phi^{\prime}(\pi)=0$, hence $\alpha_{0}=0$ and $\alpha_{1}=\pi$ are the stationary points of the $\alpha$-integral. For large $|k|$ we thus obtain

$$
\begin{aligned}
\mathcal{I} \approx & \sqrt{\frac{2 \pi}{\left|k \phi^{\prime \prime}\left(\alpha_{0}\right)\right|}} \sqrt{\frac{2 \pi}{\left|k \cos \alpha_{0}\right|\left|\mathbf{x}_{0}\right|}} \exp \left\{i\left(k \phi\left(\alpha_{0}\right)+\left(\mu_{0}+\nu_{0}\right) \pi / 4\right)\right\} u\left(k, \alpha_{0}, \pi / 2\right) \\
& +\sqrt{\frac{2 \pi}{\left|k \phi^{\prime \prime}\left(\alpha_{1}\right)\right|}} \sqrt{\frac{2 \pi}{\left|k \cos \alpha_{1}\right|\left|\mathbf{x}_{0}\right|}} \exp \left\{i\left(k \phi\left(\alpha_{1}\right)+\left(\mu_{1}+\nu_{1}\right) \pi / 4\right)\right\} u\left(k, \alpha_{1}, \pi / 2\right)
\end{aligned}
$$

with $\mu_{0}=\nu_{0}=\operatorname{sign}\left(-k \cos \alpha_{0}\right)=-\operatorname{sign}(k)$ and $\mu_{1}=\nu_{1}=\operatorname{sign}\left(-k \cos \alpha_{1}\right)=\operatorname{sign}(k)$. Hence,

$$
\mathcal{I} \approx \frac{2 \pi}{i k\left|\mathbf{x}_{0}\right|}\left[\exp \left(i k\left|\mathbf{x}_{0}\right|\right) u\left(\mathbf{k}_{0}\right)-\exp \left(-i k\left|\mathbf{x}_{0}\right|\right) u\left(-\mathbf{k}_{0}\right)\right]
$$

where $\mathbf{k}_{0}=k \mathbf{x}_{0} /\left|\mathbf{x}_{0}\right|$.

A.2. $2 \mathrm{D}$ situation. For the $2 \mathrm{D}$ situation the $\beta$-integral is omitted, hence

$$
\begin{aligned}
\mathcal{I} \approx & \sqrt{\frac{2 \pi}{\left|k \phi^{\prime \prime}\left(\alpha_{0}\right)\right|}} \exp \left\{i\left(k \phi\left(\alpha_{0}\right)-\mu \pi / 4\right)\right\} u\left(k, \alpha_{0}\right) \\
& +\sqrt{\frac{2 \pi}{\left|k \phi^{\prime \prime}\left(\alpha_{1}\right)\right|}} \exp \left\{i\left(k \phi\left(\alpha_{1}\right)+\mu \pi / 4\right)\right\} u\left(k, \alpha_{1}\right)
\end{aligned}
$$

with $\mu=\operatorname{sign}(k)$, or

$$
\mathcal{I} \approx-i \mu \sqrt{\frac{2 \pi}{|k|\left|\mathbf{x}_{0}\right|}}\left[\exp \left\{i\left(k\left|\mathbf{x}_{0}\right|+\mu \pi / 4\right)\right\} u\left(\mathbf{k}_{0}\right)-\exp \left\{-i\left(k\left|\mathbf{x}_{0}\right|+\mu \pi / 4\right)\right\} u\left(-\mathbf{k}_{0}\right)\right] .
$$

Acknowledgments. We thank three anonymous reviewers for their constructive comments, which helped us to improve the paper.

Copyright (C) by SIAM. Unauthorized reproduction of this article is prohibited. 


\section{REFERENCES}

[1] R. L. WeAVER AND O. I. LoBkis, Ultrasonics without a source: Thermal fluctuation correlations at MHz frequencies, Phys. Rev. Lett., 87 (2001), 134301.

[2] K. Wapenaar, D. Draganov, J. Thorbecke, and J. Fokkema, Theory of acoustic daylight imaging revisited, in SEG Technical Program Expanded Abstracts 2002, Society of Exploration Geophysicists, Tulsa, OK, 2002, pp. 2269-2272.

[3] A. Derode, E. Larose, M. Campillo, and M. Fink, How to estimate the Green's function of a heterogeneous medium between two passive sensors? Application to acoustic waves, Appl. Phys. Lett., 83 (2003), pp. 3054-3056.

[4] K. WAPENAAR, Retrieving the elastodynamic Green's function of an arbitrary inhomogeneous medium by cross correlation, Phys. Rev. Lett., 93 (2004), 254301.

[5] R. SNiEder, Extracting the Green's function from the correlation of coda waves: A derivation based on stationary phase, Phys. Rev. E, 69 (2004), 046610.

[6] O. A. Godin, Emergence of the acoustic Green's function from thermal noise, J. Acoustical Soc. Amer., 121 (2007), pp. EL96-EL102.

[7] R. L. Weaver And O. I. Lobkis, On the emergence of the Green's function in the correlations of a diffuse field: Pulse-echo using thermal phonons, Ultrasonics, 40 (2002), pp. 435-439.

[8] N. M. Shapiro AND M. CAmpillo, Emergence of broadband Rayleigh waves from correlations of the ambient seismic noise, Geophys. Res. Lett., 31 (2004), L07614.

[9] K. G. Sabra, P. Gerstoft, P. Roux, W. A. Kuperman, and M. C. Fehler, Extracting time-domain Green's function estimates from ambient seismic noise, Geophys. Res. Lett., 32 (2005), L03310.

[10] C. Sens-Schönfelder AND U. Wegler, Passive image interferometry and seasonal variations of seismic velocities at Merapi Volcano, Indonesia, Geophys. Res. Lett., 33 (2006), L21302.

[11] A. Curtis, H. Nicolson, D. Halliday, J. Trampert, and B. Baptie, Virtual seismometers in the subsurface of the Earth from seismic interferometry, Nature Geosci., 2 (2009), pp. 700-704.

[12] D. Draganov, K. Wapenaar, W. Mulder, J. Singer, and A. Verdel, Retrieval of reflections from seismic background-noise measurements, Geophys. Res. Lett., 34 (2007), p. L04305.

[13] P. Roux AND M. Fink, Green's function estimation using secondary sources in a shallow water environment, J. Acoustical Soc. Amer., 113 (2003), pp. 1406-1416.

[14] L. A. Brooks And P. Gerstoft, Ocean acoustic interferometry, J. Acoustical Soc. Amer., 121 (2007), pp. 3377-3385.

[15] K. G. Sabra, S. Conti, P. Roux, and W. A. Kuperman, Passive in vivo elastography from skeletal muscle noise, Appl. Phys. Lett., 90 (2007), 194101.

[16] E. Larose, L. Margerin, A. Derode, B. van Tiggelen, M. Campillo, N. Shapiro, A. Paul, L. Stehly, And M. Tanter, Correlation of random wave fields: An interdisciplinary review, Geophys., 71 (2006), pp. SI11-SI21.

[17] G. T. Schuster, Seismic Interferometry, Cambridge University Press, Cambridge, UK, 2009.

[18] R. Snieder, M. Miyazawa, E. Slob, I. Vasconcelos, and K. WapenaAr, A comparison of strategies for seismic interferometry, Surveys Geophys., 30 (2009), pp. 503-523.

[19] K. Wapenaar, D. Draganov, R. Snieder, X. Campman, and A. Verdel, Tutorial on seismic interferometry: Part 1-Basic principles and applications, Geophys., 75 (2010), pp. 75A195-75A209.

[20] H. Yao, R. D. van der Hilst, and M. V. DE Hoop, Surface-wave array tomography in SE Tibet from ambient seismic noise and two-station analysis I. Phase velocity maps, Geophys. J. Internat., 166 (2006), pp. $732-744$.

[21] C. Liang and C. A. Langston, Ambient seismic noise tomography and structure of eastern North America, J. Geophys. Res.- - Solid Earth, 113 (2008), B03309.

[22] M. Picozzi, S. Parolai, D. Bindi, And A. Strollo, Characterization of shallow geology by highfrequency seismic noise tomography, Geophys. J. Internat., 176 (2009), pp. 164-174.

[23] S. DE RIDDER AND B. BIONDI, Low frequency passive seismic interferometry for land data, in SEG Technical Program Expanded Abstracts 2010, Society of Exploration Geophysicists, Tulsa OK, 2010, pp. 4041-4046.

Copyright (C) by SIAM. Unauthorized reproduction of this article is prohibited. 
[24] M. J. Buckingham, On the two-point cross-correlation function of anisotropic, spatially homogeneous ambient noise in the ocean and its relationship to the Green's function, J. Acoustical Soc. Amer., 129 (2011), pp. 3562-3576.

[25] P. Roux, W. A. Kuperman, And K. G. Sabra, Ocean acoustic noise and passive coherent array processing, Comptes Rendus Geosci., 343 (2011), pp. 533-547.

[26] L. Stehly, M. Campillo, B. Froment, and R. L. Weaver, Reconstructing Green's function by correlation of the coda of the correlation $\left(C^{3}\right)$ of ambient seismic noise, J. Geophys. Res., 113 (2008), B11306.

[27] J. Garnier and G. Papanicolaou, Passive sensor imaging using cross correlations of noisy signals in a scattering medium, SIAM J. Imaging Sci., 2 (2009), pp. 396-437.

[28] J. Garnier and G. Papanicolaou, Fluctuation theory of ambient noise imaging, C. R. Geosci., 343 (2011), pp. 502-511.

[29] A. Curtis and D. Halliday, Directional balancing for seismic and general wavefield interferometry, Geophys., 75 (2010), pp. SA1-SA14.

[30] K. Wapenaar, J. van der Neut, E. Ruigrok, D. Draganov, J. Hunziker, E. Slob, J. Thorbecke, AND R. SNIEDER, Seismic interferometry by crosscorrelation and by multidimensional deconvolution: A systematic comparison, Geophys. J. Internat., 185 (2011), pp. 1335-1364.

[31] R. Glauber and V. Schomaker, The theory of electron diffraction, Phys. Rev., 89 (1953), pp. 667-671.

[32] K. WapenaAr, E. Slob, And R. Snieder, On seismic interferometry, the generalized optical theorem, and the scattering matrix of a point scatterer, Geophys., 75 (2010), pp. SA27-SA35.

[33] W. Heisenberg, Die "beobachtbaren Größen" in der Theorie der Elementarteilchen, Z. Physik, 120 (1943), pp. 513-538.

[34] R. G. Newton, Optical theorem and beyond, Amer. J. Phys., 44 (1976), pp. 639-642.

[35] R. Snieder, F. J. SÁnchez-Sesma, And K. WAPEnAar, Field fluctuations, imaging with backscattered waves, a generalized energy theorem, and the optical theorem, SIAM J. Imaging Sci., 2 (2009), pp. 763 776.

[36] R. Snieder, K. van Wijk, M. Haney, and R. Calvert, Cancellation of spurious arrivals in Green's function extraction and the generalized optical theorem, Phys. Rev. E, 78 (2008), 036606.

[37] D. Halliday and A. Curtis, Generalized optical theorem for surface waves and layered media, Phys. Rev. E, 79 (2009), 056603.

[38] E. J. CAndÈs And B. REcht, Exact matrix completion via convex optimization, Found. Comput. Math., 9 (2009), pp. $717-772$.

[39] E. J. CANDÈs AND T. TAO, The power of convex relaxation: Near-optimal matrix completion, IEEE Trans. Inform. Theory, 56 (2010), pp. 2053-2080.

[40] R. H. Keshavan, A. Montanari, And S. Оh, Matrix completion from noisy entries, J. Mach. Learn. Res., 11 (2010), pp. 2057-2078.

[41] R. Mazumder, T. Hastie, and R. Tibshirani, Spectral regularization algorithms for learning large incomplete matrices, J. Mach. Learn. Res., 11 (2010), pp. 2287-2322.

[42] A. Erdély, Asymptotic Expansions, Dover Publications, New York, 1956.

[43] N. Bleistein and R. A. Handelsman, Asymptotic Expansions of Integrals, Dover Publications, New York, 1975.

Copyright (C) by SIAM. Unauthorized reproduction of this article is prohibited. 